\title{
Sarcomatoid urothelial carcinoma arising in the female urethral diverticulum
}

\author{
Heae Surng Park \\ Department of Pathology, Ewha Womans University Seoul Hospital, Seoul, Korea
}

\begin{abstract}
A sarcomatoid variant of urothelial carcinoma in the female urethral diverticulum has not been reported previously. A 66-year-old woman suffering from dysuria presented with a huge urethral mass invading the urinary bladder and vagina. Histopathological examination of the resected specimen revealed predominantly undifferentiated pleomorphic sarcoma with sclerosis. Only a small portion of conventional urothelial carcinoma was identified around the urethral diverticulum, which contained glandular epithelium and villous adenoma. The patient showed rapid systemic recurrence and resistance to immune checkpoint inhibitor therapy despite high expression of programmed cell death ligand-1. We report the first case of urethral diverticular carcinoma with sarcomatoid features.
\end{abstract}

Key Words: Sarcomatoid carcinoma; Urothelial carcinoma; Urethral diverticulum

Received: March 9, 2021 Revised: April 16, 2021 Accepted: April 23, 2021

Corresponding Author: Heae Surng Park, MD, PhD, Department of Pathology, Ewha Womans University Seoul Hospital, Ewha Womans University College of Medicine, 260 Gonghang-daero, Gangseo-gu, Seoul 07804, Korea

Tel: +82-2-6986-5253, Fax: +82-2-6986-3423, E-mail: turtle98p@naver.com

Urethral diverticular carcinoma (UDC) is extremely rare; the most common histological subtype is adenocarcinoma $[1,2]$. Sarcomatoid urothelial carcinoma (UC) is also unusual. Due to the scarcity of UDC and sarcomatoid UC, related studies have been limited to small series and single case reports. Herein, we report the first case of sarcomatoid UC of the female urethral diverticulum and her treatment response to immune checkpoint inhibitor therapy.

\section{CASE REPORT}

A 66-year-old woman with dysuria was referred to the urology department after visiting the emergency room and undergoing several urinary catheterization procedures. She was hospitalized for a cerebral infarction 7 months prior and had a history of cholecystectomy owing to acute cholecystitis 6 months before. A urethral diverticulum was identified through abdominopelvic computed tomography (CT) performed during admission for cholecystectomy (Fig. 1A), although no urological evaluation was conducted at that time. On presentation, abdominopelvic CT revealed a large mass involving the urethra, posterior wall of the urinary bladder, and vagina with enlarged lymph nodes at both femoral, both inguinal, and both internal and external iliac areas (Fig. 1B). Ultrasound-guided transvaginal core needle biopsy of the urethral mass was performed, and histopathological examination revealed unspecified spindle cell sarcoma with stromal sclerosis. The patient underwent anterior pelvic exenteration with pelvic lymph node dissection.

The surgical specimen showed a 10-cm-sized, hard white mass in the urethra that had invaded the uterine cervix, anterior wall of the vagina, posterior wall of the urinary bladder, and perivesical fat (Fig. 2A). The cut surface of the mass showed central necrosis with cystic space formation. Histopathologically, the tumor was predominantly composed of pleomorphic spindle cells with moderate to severe nuclear atypia, brisk mitosis, and necrosis (Fig. 2B). The tumor nuclei were oval shaped and had vesicular chromatin and prominent nucleoli. The tumor occasionally produced abundant collagen, but neither specific mesenchymal differentiation nor a heterologous component was observed. Intratumoral lymphoplasmacytic infiltration was noted. The cystic space was focally lined by urothelial or glandular epithelium (Fig. 2C) and associated villous adenoma (Fig. 2D). 

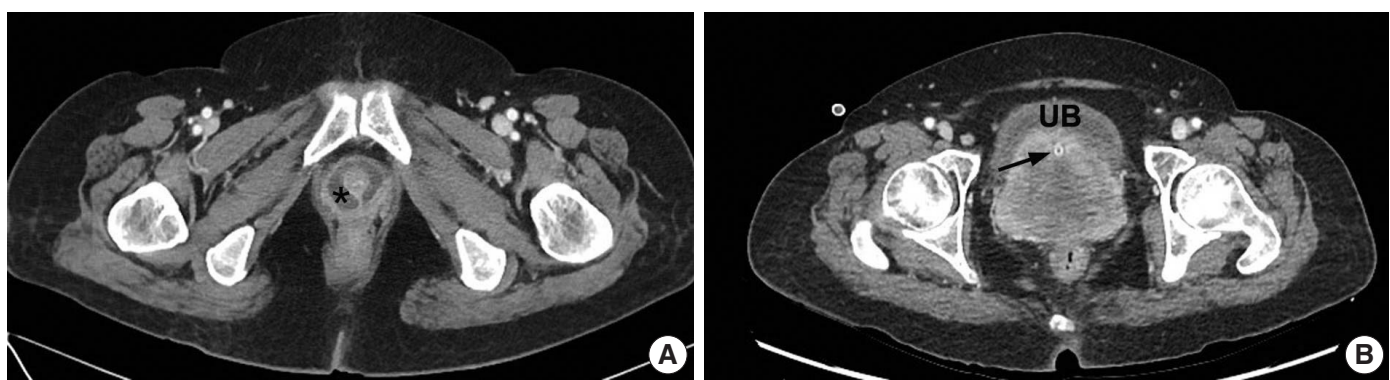

Fig. 1. Enhanced abdominopelvic computed tomography. (A) Axial image taken 7 months prior to presentation showed a urethral diverticulum (asterisk) at the level of the symphysis pubis. (B) Preoperative image revealed a large urethral mass (UB, urinary bladder; arrow, urinary catheter within the urethra).
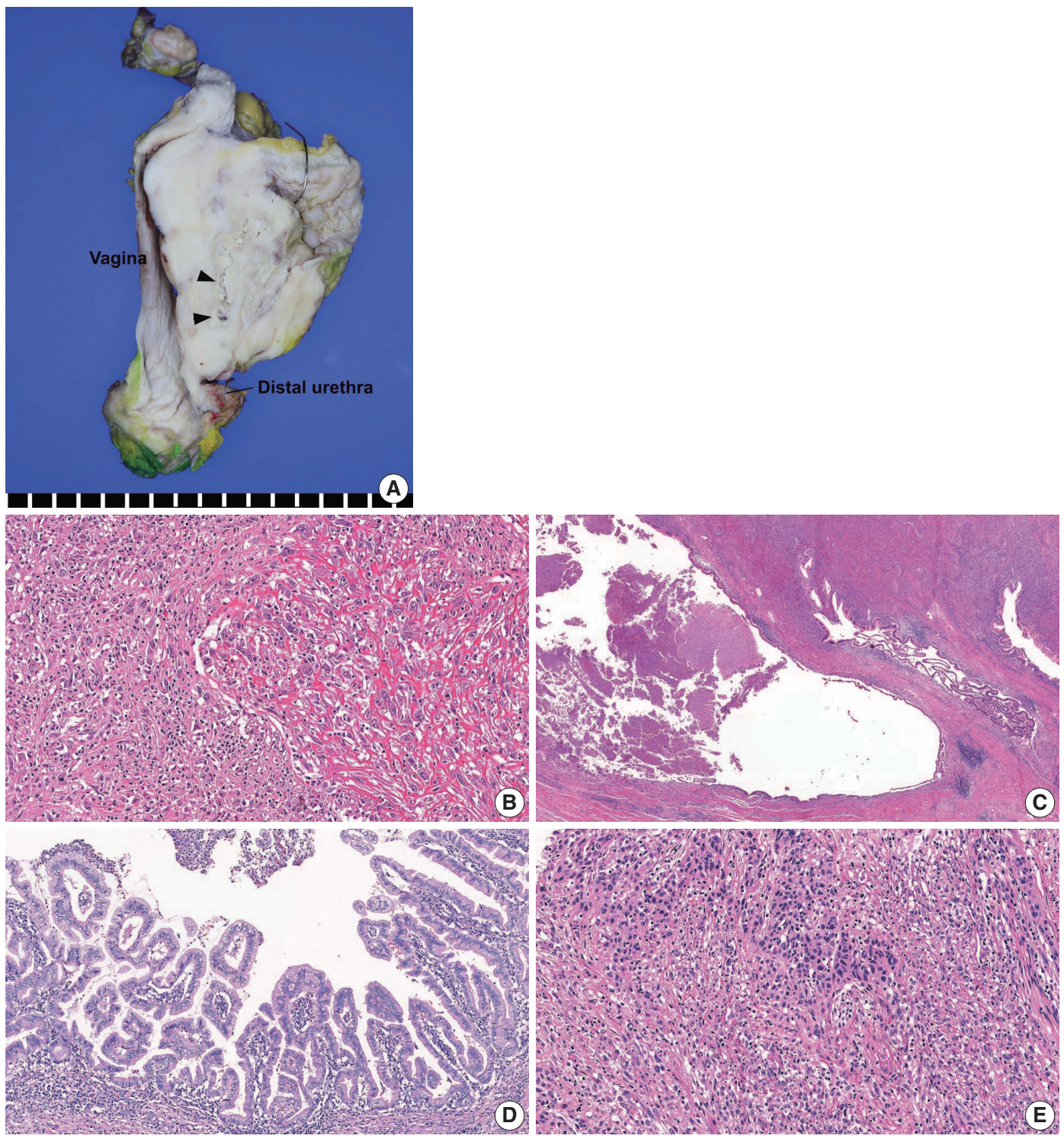

Fig. 2. Histopathological findings. (A) Gross examination revealed a 10-cm-sized, hard white urethral mass invading the uterus, vagina, urinary bladder, and perivesical fat. The cut surface showed necrosis and cystic space (arrowheads). (B) Microscopically, the majority of the tumor was composed of pleomorphic spindle cells with occasional collagen deposition. Intratumoral lymphoplasmacytic infiltration was noted. (C, D) The cystic space was lined focally by glandular epithelium (C) and associated villous adenoma (D). (E) A conventional urothelial carcinoma component was minimally present, and areas of epithelial-to-mesenchymal transition were noted. 

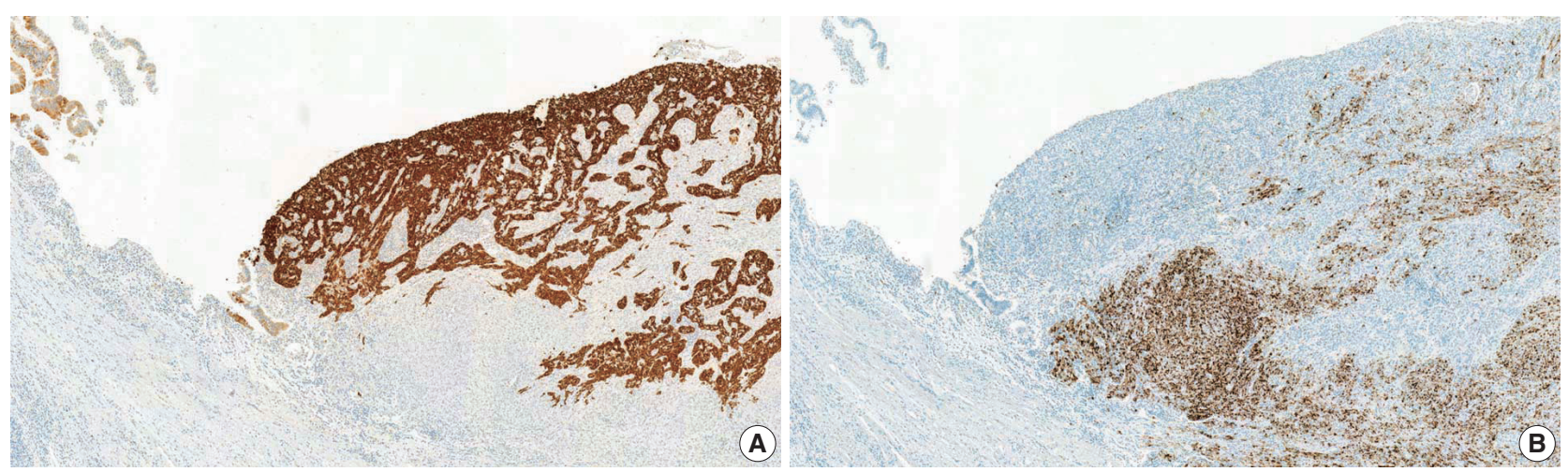

Fig. 3. Results of immunohistochemical staining. (A) Immunostaining for cytokeratin 7 highlighted the glandular epithelium (left side) and urothelial carcinoma component, whereas there was no staining of the sarcoma component. (B) Programmed death ligand-1 SP142 (Ventana Medical Systems, Tucson, AZ, USA) immunostaining showed diffuse positivity (90\%) in tumor-infiltrating immune cells (ICs) of the sarcoma component, while ICs of the carcinoma component were negative.

The lining epithelium and premalignant lesion were juxtaposed with tumor tissue, suggesting tumor formation in the urethral diverticulum. A minimal conventional urothelial carcinoma component was present (less than 1\%) near the diverticulum, and areas of epithelial-to-mesenchymal transition were noted (Fig. 2E). Although lymphovascular invasion was present, nodal metastasis was not identified among the 30 pelvic lymph nodes. Immunohistochemical staining for cytokeratin (CK) 7 highlighted the glandular epithelium and invasive carcinoma component (Fig. 3A). The invasive carcinoma component was positive for high molecular weight $\mathrm{CK}, \mathrm{p} 63$, and GATA3, indicating a urothelial nature. Urothelial carcinoma in situ was not identified. Spindle cell component was negative for panCK, CK7, GATA3, p63, smooth muscle actin, myoglobin, anaplastic lymphoma kinase (clone, 5A4), S-100, and human melanoma black-45 immunostaining, but positive for vimentin. Programmed death ligand-1 (PD-L1) SP142 (Ventana Medical Systems, Tucson, AZ, USA) immunohistochemistry showed diffuse positivity (90\%) in tumor-infiltrating immune cells (IC) of the sarcoma component, while no positive ICs were identified in the urothelial carcinoma component (Fig 3B). PD-L1 immunostaining was performed using three different tissue sections and the results were similar. The final pathological diagnosis was sarcomatoid UC arising from the urethral diverticulum (pT4N0).

During follow-up, multiple newly developed lung nodules were detected on chest CT 49 days postoperative. The patient underwent one cycle of palliative chemotherapy (adriamycin and cisplatin), but showed intolerance to the chemotherapeutic agent, and the disease progressed. She next underwent four cycles of atezolizumab and radiation therapy, but the disease continued to progress. She then received gemcitabine plus cisplatin, and the tumor showed a partial response, but tumor progresssion occurred after 4 months of treatment. The patient next received weekly paclitaxel for 2 months and exhibited stable disease. Recently, she stopped chemotherapy temporarily and is alive 24 months postoperative.

\section{DISCUSSION}

Primary female urethral carcinomas are rare, and the most common histological subtype is urothelial carcinoma (45\%), followed by adenocarcinoma (29\%), squamous cell carcinoma (19\%), and undifferentiated carcinoma (6\%), according to a national urethral cancer survey conducted in the Netherlands [3]. UDC is very unusual, accounting for only $5 \%$ of all female urethral carcinomas [1,2]. More than 120 cases of UDC have been reported, and adenocarcinoma is the most common pathology (75\%), while urothelial carcinoma (15\%) and squamous cell carcinoma $(10 \%)$ are less frequent $[1,2]$.

UDC is hypothesized to arise from a periurethral gland or metaplastic epithelium with chronic irritation, and Gartner or mesonephric duct remnants [2]. UDC might contain premalignant lesions such as villous adenoma, intestinal metaplasia, or highgrade dysplasia [4]. The present case is suggestive of tumor origination from metaplastic change because of its relation to the glandular epithelium as well as villous adenoma.

The sarcomatoid variant of urothelial carcinoma is rare, but has been reported in the urinary bladder [5], bladder diverticulum [6], ureter [7], renal pelvis [8], and urethra [9]. As far as we know, this is the first case report of sarcomatoid UC arising from a urethral diverticulum. One meta-analysis showed that sarcomatoid urothelial carcinoma of the bladder tended to present as more 
advanced disease than conventional urothelial carcinoma, which might lead to worse survival outcomes [5]. Sarcomatoid urothelial carcinoma is defined as a biphasic tumor composed of both malignant epithelial and mesenchymal elements, and the latter element is morphologically indistinguishable from sarcoma $[10,11]$. For most cases, the carcinomatous component is urothelial, but variable degrees of squamous cell carcinoma, adenocarcinoma, and small cell carcinoma components can be present $[10,11]$. The sarcomatous component is reported to constitute $20 \%$ to $100 \%$ of the tumor volume [12] and usually presents as undifferentiated high-grade sarcoma [11]. A heterologous component can be seen, most commonly osteosarcoma, followed by chondrosarcoma, leiomyosarcoma, rhabdomyosarcoma, liposarcoma, and angiosarcoma $[10,11]$. In tumors in the urinary system and those exclusively consisting of malignant spindle cells, the first differential diagnostic consideration should be sarcomatoid UC, since it can show a wide range of morphologies, and primary sarcoma of the urinary tract is rare. In such cases, extensive gross examination and immunohistochemistry must be performed to confirm the existence of a carcinoma component. In this case, transvaginal needle biopsy showed only high-grade sarcoma, suggesting primary urethral sarcoma. However, a very small urothelial carcinoma component was identified in the surgically resected specimen after extensive tissue examination.

Recently, immune checkpoint inhibitor therapy has demonstrated anti-tumor effects in advanced urothelial carcinoma. Atezolizumab is a Food and Drug Administration (FDA)-approved anti-programmed death-1/PD-L1 agent used for the treatment of advanced bladder cancer. PD-L1 SP142 immunohistochemistry was approved by the FDA as a companion diagnostic test in urothelial carcinoma patients based on the results of a phase 2 clinical trial, IMvigor210 [13]. However, a phase 3 randomized controlled trial, IMvigor211 [14], revealed higher PD-L1 SP142 expression ( $\geq 5 \%$ positivity in ICs) to be associated with response to both atezolizumab and chemotherapy in patients with platinum-treated locally advanced or metastatic urothelial carcinoma. Li et al. [15] evaluated PD-L1 SP142 expression in bladder UC and showed that the sarcomatoid variant was significantly associated with higher PD-L1 expression. In our case, the UDC showed high PD-L1 SP142 expression (IC 90\%), but the tumor progressed despite atezolizumab administration. The PD-L1-negative urothelial carcinoma component could be the driver of progression in this patient, although the progressed lesion was not confirmed histologically. We report a very unusual case of sarcomatoid UC in the female urethral diverticulum, which showed aggressive behavior and resistance to atezolizumab therapy de- spite high PD-L1 expression. Consideration of a broad range of histologic features is needed to diagnose sarcomatoid UC of the urinary system.

\section{Ethics Statement}

This study was approved by the Institutional Review Board (IRB) of Ewha Womans University Seoul Hospital (IRB No. 2021-02-23) and the need for informed consent was waived.

\section{Availability of Data and Material}

All data generated or analyzed during the study are included in this published article.

\section{Code Availability}

Not applicable.

\section{ORCID}

Heae Surng Park https://orcid.org/0000-0003-1849-5120

\section{Conflicts of Interest}

The author declare that they has no potential conflicts of interest.

\section{Funding Statement}

No funding to declare.

\section{References}

1. Ahmed K, Dasgupta R, Vats A, et al. Urethral diverticular carcinoma: an overview of current trends in diagnosis and management. Int Urol Nephrol 2010; 42: 331-41.

2. O'Connor E, Iatropoulou D, Hashimoto S, Takahashi S, Ho DH, Greenwell T. Urethral diverticulum carcinoma in females: a case series and review of the English and Japanese literature. Transl Androl Urol 2018; 7: 703-29.

3. Derksen JW, Visser O, de la Riviere GB, Meuleman EJ, Heldeweg EA, Lagerveld BW. Primary urethral carcinoma in females: an epidemiologic study on demographical factors, histological types, tumour stage and survival. World J Urol 2013; 31: 147-53.

4. Rajan N, Tucci P, Mallouh C, Choudhury M. Carcinoma in female urethral diverticulum: case reports and review of management. J Urol 1993; 150: 1911-4.

5. Gu L, Ai Q, Cheng Q, et al. Sarcomatoid variant urothelial carcinoma of the bladder: a systematic review and meta-analysis of the clinicopathological features and survival outcomes. Cancer Cell Int 2020; 20: 550 .

6. Lembo F, Subba E, Lagana AS, Vitale SG, Valenti G, Magno C. Intradiverticular sarcomatoid carcinoma of the bladder: an overview starting from a peculiar case. Urol J 2016; 13: 2800-2.

7. Wang Y, Liu H, Wang P. Primary sarcomatoid urothelial carcinoma of the ureter: a case report and review of the literature. World J Surg Oncol 2018; 16: 77.

8. Rashid S, Akhtar M. Sarcomatoid variant of urothelial carcinoma of the renal pelvis with inferior vena cava tumour thrombus: a case report and literature review. Case Rep Pathol 2018; 2018: 1837510.

9. D’Arrigo L, Costa A, Fraggetta F, Pennisi M, Pepe P, Aragona F. Carcinosarcoma of the female urethra. Urol Int 2016; 96: 370-2.

10. Moch H, Humphrey PA, Ulbright TM, Reuter VE. WHO classifica- 
tion of tumours of the urinary system and male genital organs. 4th ed. Lyon: International Agency for Research on Cancer, 2016; 92.

11. Bostwick DG, Cheng L. Urologic surgical pathology. 3rd ed. Philadelphia: Saunders-Elsevier, 2014; 298-9.

12. Sanfrancesco J, McKenney JK, Leivo MZ, Gupta S, Elson P, Hansel DE. Sarcomatoid urothelial carcinoma of the bladder: analysis of 28 cases with emphasis on clinicopathologic features and markers of epithelial-to-mesenchymal transition. Arch Pathol Lab Med 2016; 140: 543-51.

13. Balar AV, Galsky MD, Rosenberg JE, et al. Atezolizumab as first-line treatment in cisplatin-ineligible patients with locally advanced and metastatic urothelial carcinoma: a single-arm, multicentre, phase 2 trial. Lancet 2017; 389: 67-76.

14. Powles T, Duran I, van der Heijden MS, et al. Atezolizumab versus chemotherapy in patients with platinum-treated locally advanced or metastatic urothelial carcinoma (IMvigor211): a multicentre, openlabel, phase 3 randomised controlled trial. Lancet 2018; 391: 748-57.

15. Li H, Zhang Q, Shuman L, et al. Evaluation of PD-L1 and other immune markers in bladder urothelial carcinoma stratified by histologic variants and molecular subtypes. Sci Rep 2020; 10: 1439. 